Fetal Diagnosis and Therapy
Fetal Diagn Ther 2019;45:184-191

DOI: $10.1159 / 000488280$
Received: November 7, 2017

Accepted after revision: March 7, 2018

Published online: May 4, 2018

\title{
Perinatal Outcome and Long-Term Neurodevelopment after Intracranial Haemorrhage due to Fetal and Neonatal Alloimmune Thrombocytopenia
}

\author{
Dian Winkelhorst ${ }^{a}$ Marije M. Kamphuis ${ }^{a}$ Sylke J. Steggerda ${ }^{b}$ Monique Rijken ${ }^{b}$ \\ Dick Oepkes $^{\mathrm{a}}$ Enrico Lopriore ${ }^{\mathrm{b}}$ Jeanine M.M. van Klink ${ }^{\mathrm{b}}$ \\ a Department of Obstetrics, Leiden University Medical Centre, Leiden, The Netherlands; ${ }^{b}$ Division of Neonatology, \\ Department of Paediatrics, Leiden University Medical Centre, Leiden, The Netherlands
}

\section{Keywords}

Fetal and neonatal alloimmune thrombocytopenia .

Intracranial haemorrhage $\cdot$ Long-term outcome .

Neurodevelopment

\begin{abstract}
Objectives: To evaluate the perinatal and long-term neurodevelopmental outcome in a cohort of children with intracranial haemorrhage $(\mathrm{ICH})$ due to fetal and neonatal alloimmune thrombocytopenia (FNAIT) and to clearly outline the burden of this disease. Subjects and Methods: We performed an observational cohort study and included all consecutive cases of ICH caused by FNAIT from 1993 to 2015 at Leiden University Medical Centre. Neurological, motor, and cognitive development were assessed at a minimum age of 1 year. The primary outcome was adverse outcome, defined as perinatal death or severe neurodevelopmental impairment (NDI). Severe NDI was defined as any of the following: cerebral palsy (Gross Motor Function Classification System [GMFCS] level $\geq I I)$, bilateral deafness, blindness, or severe motor and/or cognitive developmental delay ( $<-2$ SD). $\boldsymbol{R e}$ sults: In total, 21 cases of ICH due to FNAIT were included in the study. The perinatal mortality rate was 10/21 (48\%).
\end{abstract}

Long-term outcome was assessed in 10 children ( $n=1$ lost to follow-up). Severe and moderate NDI were diagnosed in $6 / 10(60 \%)$ and $1 / 10(10 \%)$ of the surviving children. The overall adverse outcome, including perinatal mortality or severe NDI, was 16/20 (80\%). Conclusions: The risk of perinatal death or severe NDI in children with ICH due to FNAIT is high. Only screening and effective preventive treatment can avoid this burden.

(c) 2018 The Author(s)

Published by S. Karger AG, Basel

\section{Introduction}

Fetal and neonatal alloimmune thrombocytopenia (FNAIT) is one of the leading causes of thrombocytopenia in otherwise healthy newborns [1,2]. Maternal alloantibodies are formed after exposure to the incompatible, paternally derived human platelet antigen (HPA) on fetal platelets. In FNAIT, these alloantibodies are predominantly targeted against HPA-1a, in approximately $80 \%$ of cases $[3,4]$. When these antibodies enter the fetal circula-

D. Winkelhorst and M.M. Kamphuis contributed equally to this work. 
tion, they can destroy fetal platelets and damage endothelial cells [5]. Hence, FNAIT presents as an (asymptomatic) thrombocytopenia or results in bleeding complications. The most feared complication is intracranial haemorrhage (ICH), due to its associated risk of lifelong handicaps and neurological sequelae $[6,7]$. In addition, ICHs caused by FNAIT have a high recurrence rate, in up to $79 \%$ of subsequent pregnancies [8]. Therefore, in the absence of population-based screening for FNAIT, current management is focused on preventing the occurrence of bleeding complications and ICHs in subsequent pregnancies through antenatal treatment with intravenous immunoglobulin (IVIG) [9]. ICH is estimated to occur in 1 out of 25,000 pregnancies and in 1 out of 10 cases of severe FNAIT [10].

Despite the fact that ICH caused by FNAIT is often more severe compared to ICH from other causes, leading to a high mortality and handicap rate, no detailed longterm follow-up studies have been published so far $[8,11$, 12]. Long-term follow-up data are necessary for performing adequate evidence-based counselling of parents and for professionals involved in guiding these children. Even more, in light of potential future implementation of population-based screening, knowledge on the long-term implications of these ICHs is indispensable.

We evaluated the perinatal and long-term neurodevelopmental outcome in a cohort of children with ICH due to FNAIT and clearly outlined the burden of this disease in survivors in the current era of fetal medicine and neonatal intensive care treatment possibilities.

\section{Subjects and Methods}

\section{Study Population}

The Leiden University Medical Centre serves as the national centre of expertise for FNAIT in the Netherlands. From 1993 to 2015, all consecutive cases with ICH due to FNAIT were identified and eligible for inclusion. We identified through women that were counselled, diagnosed, or treated at our centre, either in the pregnancy of interest or during a subsequent pregnancy. These cases were cross-checked with Sanquin, the national reference laboratory for FNAIT, where the diagnosis of FNAIT was confirmed in case of incompatibility between maternal and paternal/fetal HPA type in combination with the detection of maternal anti-HPA antibodies.

\section{Outcomes}

The primary outcome was perinatal death and/or severe neurodevelopmental impairment (NDI). Severe NDI was defined as any of the following: severe cerebral palsy (Gross Motor Function Classification System [GMFCS] level $\geq$ II), a cognitive and/or motor test score $<70$ (<-II SD), bilateral blindness, or bilateral deafness requiring amplification. The secondary outcome was moder- ate NDI defined as cerebral palsy (GMFCS level $<$ II) or mild-tomoderate motor and/or cognitive developmental delay $(<-1 \mathrm{SD}$ and $>-2$ SD).

The following ante- and neonatal data were retrieved from the medical files: antenatal treatment, gestational age at birth, mode of delivery, birth weight, platelet count at birth, clinical course, and cerebral imaging. When available, neuroradiological images were reviewed by an experienced neonatologist (S.J.S.) to confirm the presence of ICH and to classify the type of bleeding. When original images from another hospital were unavailable for review, written reports by other experienced radiologists were obtained. Haemorrhages were classified as subdural, subarachnoid, cerebellar, intraventricular, or intraparenchymal, with a separate notion for unilateral or bilateral occurrence and the extent of lobar involvement (frontal, parietal, occipital, or temporal) [13]. Cases with no imaging or no classification of imaging performed elsewhere available were excluded.

Neurological, motor, and cognitive development was assessed at a minimum age of 1 year. The following standardised psychometric tests, appropriate for age, were used: the Bayley Scales of Infant and Toddler Development third edition (Bayley-III), the Wechsler Preschool Primary Scale of Intelligence third edition (WPPSI-III), and the Wechsler Intelligence Scale for Children third edition (WISC-III) [14-16]. Bayley-III, WPPSI-III, and WISC-III scores follow a normal distribution curve with a mean of 100 and a standard deviation of 15. A cognitive test score, i.e., a Bayley-III cognitive composite score, WPPSI-III, or WISC-III total IQ score $<70(<-2 \mathrm{SD})$, indicates severe cognitive delay. Scores $<85$ ( $<-1 \mathrm{SD}$ ) indicate mild-to-moderate cognitive delay. Children with severe cognitive impairments (with scores $<50$ ) or who were unable to participate in standardised testing due to severe cognitive impairment were assigned a score of 49 in the database. Testing was either performed by our specialised medical psychologist at our outpatient clinic or results were requested at their institution of care.

Cerebral palsy was defined according to the European Cerebral Palsy Network and classified as diplegia, hemiplegia, quadriplegia, dyskinetic, or mixed. Subsequently, cerebral palsy was scaled according the GMFCS into level I-V, varying from decreased speed, balance, and coordination at level I to impaired in all motor functions, cannot sit, stand, walk independently, and has physical impairments that restrict voluntary control of movement and the ability to maintain head and neck position against gravity at level $\mathrm{V}[17]$.

\section{Statistical Analysis}

All data were analysed with the SPSS software (V.18.0; SPSS Inc., Chicago, IL, USA) using descriptive statistics. Categorical data are presented as numbers and percentages. Continuous variables are presented as median with range or mean with standard deviation.

\section{Results}

\section{Study Population}

Between 1993 and 2015, a total of 31 cases with an ICH due to FNAIT were identified (Fig. 1). Of these, 7 (23\%) 


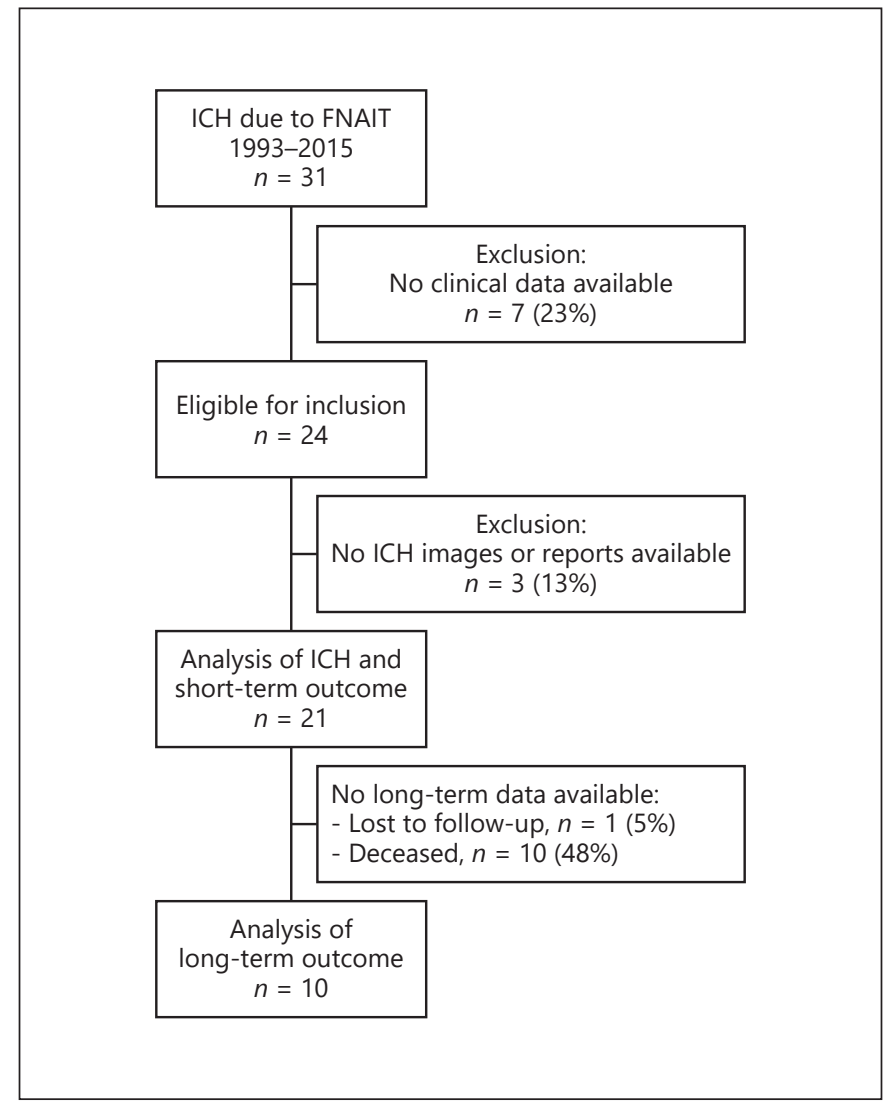

Fig. 1. Flow chart showing the derivation of the study population. FNAIT, fetal and neonatal alloimmune thrombocytopenia; ICH, intracranial haemorrhage.

were excluded because there were no clinical data available on either short- or long-term outcome. Three additional cases (13\%) were excluded because there were no images or reports of the ICH available. The remaining 21 children with ICH were included and assessed for shortterm outcome. Perinatal death was reported in 10 (48\%) cases because of fetal demise at 19-22 weeks of gestation $(n=3)$, death during labour after drainage of posthaemorrhagic hydrocephalus $(n=1)$, and neonatal death related to severe ICH $(n=6)$.

Obstetric history revealed a previous miscarriage in 10 (48\%) cases (Table 1). HPA-1a was the predominantly involved alloantibody, in 18 (86\%) cases. In 5 (24\%) cases the ICH occurred in the first pregnancy, and in 12 cases the ICH affected the first-born child. The lowest platelet count in all cases not treated antenatally with IVIG was $<30 \times 10^{9} / \mathrm{L}$, with a median of $11 \times 10^{9} / \mathrm{L}$.
Table 1. Demographic characteristics of the study population $(n=$ 21)

\begin{tabular}{lc}
\hline Maternal age, years & $30(21-41)$ \\
Obstetric history & \\
Sibling with ICH & $1(5 \%)$ \\
Sibling with FNAIT & $2(10 \%)$ \\
Miscarriage & $10(48 \%)$ \\
HPA type & $18(86 \%)$ \\
HPA-1a & $2(10 \%)$ \\
HPA-5b & $1(5 \%)$ \\
HPA-5a & $20(95 \%)$ \\
Obstetric characteristics & $5(24 \%)$ \\
Singleton pregnancy & $12(57 \%)$ \\
First pregnancy & \\
First-born child & $4(19 \%)$ \\
Antenatal treatment, IVIG $1 \mathrm{~g} / \mathrm{kg} /$ week & $36^{+0}\left(30^{+0}-41^{+6}\right)$ \\
after antenatal detection of ICH & \\
Gestational age at delivery, weeks ${ }^{1}$ & $6(33 \%)$ \\
Delivery & $3(17 \%)$ \\
Vaginal & $9(50 \%)$ \\
Ventouse & \\
Caesarean section & $13(62 \%)$ \\
Neonatal characteristics & \\
Male sex & \\
Birth weight, g & \\
Platelet count, $\times 10^{9} / \mathrm{L}^{1}$ & $11(6-29)$ \\
$\quad$ Without antenatal IVIG & \\
$\quad$ With antenatal IVIG &
\end{tabular}

FNAIT, fetal and neonatal alloimmune thrombocytopenia; HPA, human platelet antigen; ICH, intracranial haemorrhage; IVIG, intravenous immunoglobulin. ${ }^{1}$ Terminations of pregnancy excluded $(n=3)$.

\section{Short-Term Outcome}

Antenatal Treatment. In 11 (52\%) cases, the ICH was already detected antenatally, and in 4 of these pregnancies antenatal treatment was administered. One mother (\#17) had a previous child with FNAIT without ICH, which led to the proposed plan of antenatal treatment with IVIG from 28 weeks of gestation. Just before the start of treatment a haemorrhage was detected during fetal cranial ultrasound. IVIG was started as planned. The second case concerned a dichorionic twin pregnancy (\#16) of which one suffered from ICH. Maternal HPA-5b antibodies were found and IVIG was started to protect the co-twin from bleeding and to prevent worsening of bleeding of the affected fetus. In the other 2 cases, ICH was detected during routine ultrasound at 20 weeks of gestation. In one of these cases, the mother had a previous child with $\mathrm{ICH}$, presumed to be caused by birth trauma. In this subsequent pregnancy, HPA-5a antibodies were detected and
Winkelhorst/Kamphuis/Steggerda/ Rijken/Oepkes/Lopriore/van Klink 
Table 2. Intracranial haemorrhage characteristics and short-term outcome

\begin{tabular}{|c|c|c|c|c|c|c|c|}
\hline Child & GA at & Antenatal & ICH location & Associated lesions & Mortality & \multicolumn{2}{|c|}{ Obstetric history } \\
\hline 1 & $33^{+4}$ & no & $\begin{array}{l}\text { extensive subarachnoid and unilateral } \\
\text { parenchymal frontal/temporal/occipital }\end{array}$ & - & yes, neonatal & G1P0 & \\
\hline 2 & $35^{+0}$ & no & $\begin{array}{l}\text { unilateral intraventricular and } \\
\text { parenchymal }\end{array}$ & hydrocephalus & yes, neonatal & G2P1 & healthy child \\
\hline 4 & $36^{+5}$ & no & extensive bilateral parenchymal & & yes, neonatal & G3P1 & healthy child, miscarriage \\
\hline 5 & $38^{+1}$ & no & extensive bilateral parenchymal & hydrocephalus & yes, fetal & G2P0 & miscarriage \\
\hline 6 & $22^{+0}$ & no & bilateral parenchymal & hydrocephalus & yes, TOP & G2P0 & miscarriage \\
\hline 7 & $32^{+2}$ & no & extensive subarachnoid & & yes, neonatal & G2P1 & child with trisomy 21 \\
\hline 10 & $19^{+4}$ & no & $\begin{array}{l}\text { unilateral parenchymal and } \\
\text { intraventricular }\end{array}$ & - & yes, TOP & G3P1 & healthy child, miscarriage \\
\hline 11 & $38^{+1}$ & no & unilateral parenchymal, occipital & - & no & G3P0 & two miscarriages \\
\hline 12 & $36^{+0}$ & no & unilateral parenchymal, temporal & hydrocephalus, VPD & no & G1P0 & \\
\hline 13 & $35^{+0}$ & no & bilateral parenchymal, temporal & $\begin{array}{l}\text { porencephalic cyst } \\
\text { hydrocephalus, VPD }\end{array}$ & no & G2P0 & miscarriage \\
\hline 14 & $36^{+1}$ & no & $\begin{array}{l}\text { bilateral parenchymal, temporal, and } \\
\text { occipital }\end{array}$ & $\begin{array}{l}\text { porencephalic cyst } \\
\text { hydrocephalus, VPD }\end{array}$ & no & G2P0 & miscarriage \\
\hline 15 & $35^{+3}$ & $\begin{array}{l}\text { yes, from } \\
30 \text { weeks }\end{array}$ & $\begin{array}{l}\text { extensive bilateral intraventricular, } \\
\text { parenchymal, and cerebellar } \\
\text { haemorrhage }\end{array}$ & $\begin{array}{l}\text { bilateral porencephalic } \\
\text { cyst, cerebellar destruction } \\
\text { hydrocephalus, VPD }\end{array}$ & no & G2P1 & healthy child \\
\hline 19 & $37^{+6}$ & $\begin{array}{l}\text { yes, from } \\
20 \text { weeks }\end{array}$ & $\begin{array}{l}\text { unilateral parenchymal, intraventricular, } \\
\text { and bilateral cerebellar }\end{array}$ & $\begin{array}{l}\text { hydrocephalus, unilateral } \\
\text { porencephalic cyst }\end{array}$ & no & G4P2 & $\begin{array}{l}\text { immature delivery at } \\
17 \text { weeks, child with ICH, } \\
\text { TOP }\end{array}$ \\
\hline 20 & $41^{+5}$ & no & $\begin{array}{l}\text { bilateral frontal parenchymal and } \\
\text { intraventricular }\end{array}$ & $\begin{array}{l}\text { hydrocephalus, bilateral } \\
\text { porencephalic cysts }\end{array}$ & no & G2P0 & molar pregnancy \\
\hline 21 & $37^{+0}$ & no & extensive bilateral intraventricular & - & no & G2P1 & healthy child \\
\hline
\end{tabular}

FNAIT, fetal and neonatal alloimmune thrombocytopenia; G, gravidity; GA, gestational age in weeks; ICH, intracranial haemorrhage; IVIG, intravenous immunoglobulin; P, parity; TOP, termination of pregnancy; VPD, ventriculoperitoneal drain. ${ }^{1}$ ICH detected before planned start of IVIG treatment.

FNAIT was diagnosed. In 19 (90\%) cases, it was clear that the ICH occurred antenatally. In the other 2 cases, the exact timing of the ICH was not reported.

Neuroimaging Examinations of ICH. The type and localisation of ICH in the 21 children with ICH are reported in Table 2. From 8 (38\%) children, MRI images were available for review; the other 13 (62\%) could be classified using written reports. Nineteen (91\%) children had intraparenchymal haemorrhage. In 8 cases there was also intraventricular bleeding and in 2 cases subarachnoid bleed- 
Table 3. Intracranial haemorrhage and long-term outcome

\begin{tabular}{|c|c|c|c|c|c|c|c|}
\hline $\begin{array}{l}\text { Child } \\
\text { No. }\end{array}$ & Associated lesions & $\begin{array}{l}\text { Age at } \\
\text { evaluation }\end{array}$ & Cerebral palsy & $\begin{array}{l}\text { Developmental } \\
\text { test }\end{array}$ & $\begin{array}{l}\text { Total } \\
\text { IQ }\end{array}$ & Long-term outcome & $\begin{array}{l}\text { Severe } \\
\text { NDI }\end{array}$ \\
\hline 11 & none & 8 years & - & WISC-III & 86 & $\begin{array}{l}\text { attention deficit hyperactivity } \\
\text { disorder }\end{array}$ & no \\
\hline 12 & hydrocephalus, VPD & $\begin{array}{l}2,8 \text {, and } \\
14 \text { years }\end{array}$ & $\begin{array}{l}\text { spastic tetraplegia, } \\
\text { GMFCS level V }\end{array}$ & $\begin{array}{l}\text { Bayley-III, } \\
\text { Reynell-Zinkin, } \\
\text { KID-N }\end{array}$ & 49 & $\begin{array}{l}\text { bilateral blindness, severe cognitive } \\
\text { and motor delay, epilepsy }\end{array}$ & yes \\
\hline 14 & $\begin{array}{l}\text { porencephalic cyst } \\
\text { hydrocephalus, VPD }\end{array}$ & 23 years & $\begin{array}{l}\text { spastic tetraplegia, } \\
\text { GMFCS level V }\end{array}$ & $\begin{array}{l}\text { not tested due to } \\
\text { severe impairment }\end{array}$ & 49 & $\begin{array}{l}\text { bilateral blindness, hearing } \\
\text { impairment, severe cognitive and } \\
\text { motor delay }\end{array}$ & yes \\
\hline 15 & $\begin{array}{l}\text { bilateral porencephalic } \\
\text { cyst, cerebellar destruction } \\
\text { hydrocephalus, VPD }\end{array}$ & 3 years & $\begin{array}{l}\text { spastic diplegia, } \\
\text { GMFCS level IV }\end{array}$ & SON & 60 & severe cognitive and motor delay & yes \\
\hline 18 & none & 7 years & - & WISC-III & 112 & & no \\
\hline 19 & $\begin{array}{l}\text { hydrocephalus, unilateral } \\
\text { porencephalic cyst }\end{array}$ & 5 years & $\begin{array}{l}\text { spastic hemiplegia, } \\
\text { GMFCS level II }\end{array}$ & WPPSI-III & 85 & $\begin{array}{l}\text { problems with behaviour and } \\
\text { attention regulation }\end{array}$ & no \\
\hline 20 & $\begin{array}{l}\text { hydrocephalus, bilateral } \\
\text { porencephalic cysts }\end{array}$ & 8 years & $\begin{array}{l}\text { spastic diplegia, } \\
\text { GMFCS level II }\end{array}$ & SON & 50 & $\begin{array}{l}\text { severe cognitive and motor delay, } \\
\text { epilepsy }\end{array}$ & yes \\
\hline 21 & none & loss of con & $t$ information, no lo & -term follow-up ava & & & \\
\hline
\end{tabular}

Bayley-III, Bayley Scales of Infant and Toddler Development third edition; GMFCS, Gross Motor Function Classification System; KID-N, Kent Infant Development Scale; NDI, neurodevelopmental impairment; SON, Snijders-Oomen Nonverbal Intelligence Test; VPD, ventriculoperitoneal drain; WISC-III, Wechsler Intelligence Scale for Children third edition; WPPSI-III, Wechsler Preschool Primary Scale of Intelligence third edition.

ing. Eight cases had bilateral haemorrhage. Eleven cases were complicated by posthaemorrhagic hydrocephalus, of whom 6 developed a porencephalic cyst, resulting in 5 of these children requiring a ventricular peritoneal shunt.

\section{Long-Term Neurodevelopmental Outcome}

In total, 10 surviving children with ICH were included for long-term follow-up (Table 3). Long-term outcome could not be assessed in 1 child (5\%) due to loss of contact information.

Neurodevelopment had already been assessed elsewhere (rehabilitation clinic or paediatric department) in 6 cases using developmental tests adapted to the child's cognitive, motor, and/or visual impairments (i.e., Snijders-Oomen Nonverbal Intelligence Test or Kent Infant Development Scale). Two children were evaluated by the medical psychologist at our centre. Two children could not be assessed with psychometric tests due to very severe cognitive and motor impairment and were assigned a score of 49. Children were tested at a median age of 7.5 years (range 1-23). The overall adverse outcome, including perinatal mortality or NDI, was 16/20 (80\%).

Severe NDI in the studied cohort was found in $6 / 10$ cases (60\%). Cerebral palsy was diagnosed in 7 cases (70\%). One child had moderate NDI due to spastic hemiparesis, GMFCS level I. Severe cognitive delay was detected in 6 children (60\%) and severe motor delay in 6 children (60\%). Three children were blind (30\%) and 1 child was diagnosed with severe visual impairment. Epilepsy was reported in $4(40 \%)$ children. One child was diagnosed with attention deficit hyperactivity disorder; 1 child had problems with behaviour and attention regulation, but was too young to be already diagnosed with attention deficit hyperactivity disorder. 


\section{Discussion}

This study shows that ICH caused by FNAIT is associated with a high risk of perinatal death and lifelong neurological sequelae in survivors. Of the 10 surviving infants, 6 had severe NDI and 2 had moderate NDI. Therefore, only 2 of the 10 survivors were completely free of long-term neurodevelopmental sequelae. Cerebral palsy was diagnosed in $70 \%$ and severe cognitive delay in $60 \%$. In addition, $40 \%$ of the children had severe visual impairment and $40 \%$ was diagnosed with epilepsy. Our findings stress the severity and implications of major and permanent lifelong impairments associated with FNAIT, particularly in case of $\mathrm{ICH}$.

The vast majority (90\%) of the ICHs occurred antenatally, which is in line with a previously published report on the short-term outcome of 43 ICHs due to FNAIT $[7,18]$. Like in this previously reported series, most ICHs were parenchymal haemorrhages, with the majority complicated by hydrocephalus and/or porencephalic cysts [7]. In our cohort, cases with hydrocephalus and porencephalic cysts were more likely to result in severe NDI (6/7 and 5/6, respectively). Due to the relatively small sample size, no correlation could be identified between localisation (frontal/ temporal/occipital or parenchymal, intraventricular, cerebellar) or extent (uni-/bilateral) and long-term outcome.

Obviously, our study does not match the true prevalence of ICH in our country, as it is a single-centre study and there is a considerable amount of cases with missing clinical information $(7 / 31,23 \%)$. This might have resulted in an overrepresentation of the more severe cases of $\mathrm{ICH}$. For example, we report a rate of perinatal death of $48 \%$ (10/21), which is somewhat higher than the previously reported rate of $35 \%(15 / 43)$ [7]. Also, since many women were identified because they were treated or counselled at our centre in subsequent pregnancies, we might have found a higher rate of primigravid women and first-born children. Furthermore, cultural differences or legal restrictions in administration of intensive neonatal care may have influenced the outcome in this cohort. It is plausible that withholding or withdrawing neonatal intensive care treatment in cases with poor prognosis may have led to a higher perinatal mortality and therefore to a lower number of survivors with poor neurodevelopmental outcome. However, cases were not selected because of behavioural or developmental problems, so it is not likely that cases with better developmental outcome were missed. Undoubtedly, there is heterogeneity in the developmental testing performed, adapted to the age as well as to the severity of impairment of the children included for follow-up. This severity limited our ability to perform standardised psychometric testing in all children.

Adverse Outcome of ICH due to FNAIT
Despite these limitations, this is an unique study that focuses on the long-term outcome of ICH due to FNAIT, clearly outlining the burden of this disease in survivors. One of the strengths of this study is that we used standardised psychometric tests. Moreover, we were able to do long-term follow-up at a median age of 7.5 years. Previously, follow-up at 1 year had been analysed in newly detected FNAIT cases, with various clinical presentations, by Knight et al. [19]. They reported death or disability in 9 out of 88 cases, 2 infants died, 2 infants had severe global developmental delay, 4 infants had motor and visual impairment, and 1 infant had only visual problems. However, no standardised tests were reported, and the classification of impairment was not further specified. Lastly, we were able to focus on a clear and homogeneous group consisting of children with ICH solely due to FNAIT. Earlier, cohorts of ICHs have been described, the largest series of intraventricular haemorrhage in fullterm newborns being reported by Mao et al. [11]. They analysed a total of 36 newborns and found a low mortality rate and, generally, a favourable outcome, with $63 \%$ of all cases having no or only mild impairment. In contrast, they found FNAIT to be the single most important cause of adverse outcome. Out of 9 cases, 3 children died and 6 were severely impaired. Jocelyn and Casiro [12] studied a cohort of 15 intraventricular haemorrhage cases in fullterm newborns, of which three were caused by FNAIT. Of these 3 patients, 2 survived and were both severely impaired. Both studies are limited by the small number of patients as well as by their selection of cases. Whereas both studies selected newborns with a diagnosis of intraventricular haemorrhage, there might be an underrepresentation of (minor) ICHs caused by FNAIT.

In the absence of screening programs for FNAIT, the disease is almost always detected after birth of an affected child, and preventive measures with antenatal IVIG can only be taken in the following pregnancies. Implementation of routine HPA typing, primarily for HPA-1a, and consequent antibody screening in the near future would strongly reduce the burden associated with this disease. However, before such screening can be implemented, the costs and potential benefits should be weighed carefully. So far, several attempts to estimate cost-effectiveness reached the same conclusion, namely that such programs are likely to be cost-effective $[10,20,21]$. This study, as it is the first one to provide detailed long-term follow-up data of children who suffered ICH due to FNAIT, provides essential knowledge for this debate. In addition, prospective studies including general screening for FNAIT and long-term follow-up are needed to learn 
more about the pathophysiology of this disease, including establishing if there is also a milder phenotype of ICH with discrete symptoms and better outcome. Furthermore, whereas only a proportion of alloimmunised pregnancies will result in devastating ICH as described in this study, research is needed to establish diagnostic tools to identify pregnancies that are at high risk for these bleeding complications and that would benefit from antenatal intervention and treatment.

\section{Conclusion}

This is the first study focusing and reporting on the long-term neurodevelopmental outcome of children suffering from ICH caused by FNAIT, using standardised psychometric measures. In the vast majority of cases, ICH leads to either perinatal death or, in survivors, severe impairment. These long-term sequelae can only be avoided by screening and effective preventive treatment.

\section{Statement of Ethics}

This study was approved by the institutional review board of the Leiden University Medical Centre (P11.190/NL37798.058.11). All parents gave written informed consent for the follow-up of their children.

\section{Disclosure Statement}

The authors have no conflicts of interest and no financial relationships relevant to this article to disclose. No funding was obtained for this study.

\section{Author Contributions}

Marije M. Kamphuis and Dian Winkelhorst conceptualised and designed the study, carried out the initial analyses, drafted the initial manuscript, reviewed and revised the manuscript, and approved the final manuscript as submitted. Sylke J. Steggerda scored all brain imaging, contributed to the analysing of the data, critically reviewed and revised the manuscript, and approved the final manuscript as submitted. Monique Rijken and Dick Oepkes coordinated and supervised the data collection, critically reviewed and revised the manuscript, and approved the final manuscript as submitted. Enrico Lopriore conceptualised and designed the study, supervised and coordinated the data collection and analyses, supervised the drafting of the initial manuscript, critically reviewed and revised the manuscript, and approved the final manuscript as submitted. Jeanine M.M. van Klink conceptualised and designed the study, supervised, coordinated, and contributed to the data collection and analyses, supervised the drafting of the manuscript, critically reviewed and revised the manuscript, and approved the final manuscript as submitted.

\section{References}

1 Dreyfus M, Kaplan C, Verdy E, Schlegel N, Durand-Zaleski I, Tchernia G: Frequency of immune thrombocytopenia in newborns: a prospective study. Immune Thrombocytopenia Working Group. Blood 1997;89:44024406.

2 Burrows RF, Kelton JG: Fetal thrombocytopenia and its relation to maternal thrombocytopenia. N Engl J Med 1993;329:1463-1466.

3 Davoren A, Curtis BR, Aster RH, McFarland JG: Human platelet antigen-specific alloantibodies implicated in 1162 cases of neonatal alloimmune thrombocytopenia. Transfusion 2004;44:1220-1225.

4 Mueller-Eckhardt C, Kiefel V, Grubert A, Kroll H, Weisheit M, Schmidt S, Mueller-Eckhardt G, Santoso S: 348 cases of suspected neonatal alloimmune thrombocytopenia Lancet 1989;1:363-366.

5 Yougbaré I, Lang S, Yang H, Chen P, Zhao X, Tai WS, Zdravic D, Vadasz B, Li C, Piran S, Marshall A, Zhu G, Tiller H, Killie MK, Boyd S, Leong-Poi H, Wen XY, Skogen B, Adamson SL, Freedman J, Ni H: Maternal anti-platelet $\beta 3$ integrins impair angiogenesis and cause intracranial hemorrhage. J Clin Invest 2015; 125:1545-1556

6 Spencer JA, Burrows RF: Feto-maternal alloimmune thrombocytopenia: a literature review and statistical analysis. Aust NZ J Obstet Gynaecol 2001;41:45-55.

7 Tiller H, Kamphuis MM, Flodmark O, Papadogiannakis N, David AL, Sainio S, Koskinen S, Javela K, Wikman AT, Kekomaki R, Kanhai HH, Oepkes D, Husebekk A, Westgren M: Fetal intracranial haemorrhages caused by fetal and neonatal alloimmune thrombocytopenia: an observational cohort study of 43 cases from an international multicentre registry. BMJ Open 2013;3:e002490.

8 Radder CM, Brand A, Kanhai HH: Will it ever be possible to balance the risk of intracranial haemorrhage in fetal or neonatal alloimmune thrombocytopenia against the risk of treatment strategies to prevent it? Vox Sang 2003; 84:318-325.

9 Winkelhorst D, Murphy MF, Greinacher A, Shehata N, Bakchoul T, Massey E, Baker J, Lieberman L, Tanael S, Hume H, Arnold DM, Baidya S, Bertrand G, Bussel J, Kjaer M,
Kaplan C, Kjeldsen-Kragh J, Oepkes D, Ryan $\mathrm{G}$ : Antenatal management in fetal and neonatal alloimmune thrombocytopenia: a systematic review. Blood 2017;129:1538-1547.

10 Kamphuis MM, Paridaans N, Porcelijn L, De Haas M, Van Der Schoot CE, Brand A, Bonsel GJ, Oepkes D: Screening in pregnancy for fetal or neonatal alloimmune thrombocytopenia: systematic review. BJOG 2010;117:1335-1343.

11 Mao C, Guo J, Chituwo BM: Intraventricular haemorrhage and its prognosis, prevention and treatment in term infants. J Trop Pediatr 1999;45:237-240.

12 Jocelyn LJ, Casiro OG: Neurodevelopmental outcome of term infants with intraventricular hemorrhage. Am J Dis Child 1992;146:194197.

13 Inder TE, Perlman M, Volpe JJ: Intracranial hemorrhage: subdural, subarachnoid, intraventricular (term infant), miscellaneous; in Volpe JJ (ed): Neurology of the Newborn, ed 6. Philadelphia, Elsevier, 2018, pp 593-622.

14 Bayley N: Bayley Scales of Infant and Toddler Development, ed 3. San Antonio, Pearson Education, Inc., 2006. 
15 Hendriksen J, Hurks P: WPPSI-III-NL Nederlandstalige bewerking: Afname- en scoringshandleiding [Dutch version of the WPPSI-III-NL: Administration and scoring manual]. Amsterdam, Pearson Assessment and Information BV, 2009.

16 Wechsler D: WISC-III Wechsler Intelligence Scale for Children - Manual, ed 3. San Antonio, The Psychological Corporation of America, 1991.

17 Palisano R, Rosenbaum P, Walter S, Russell D, Wood E, Galuppi B: Development and reliability of a system to classify gross motor function in children with cerebral palsy. Dev Med Child Neurol 1997;39:214-223.
18 Kamphuis MM, Tiller $\mathrm{H}$, van den Akker ES, Westgren M, Tiblad E, Oepkes D: Fetal and neonatal alloimmune thrombocytopenia: management and outcome of a large international retrospective cohort. Fetal Diagn Ther 2017;41:251-257.

19 Knight M, Pierce M, Allen D, Kurinczuk JJ, Spark P, Roberts DJ, Murphy MF: The incidence and outcomes of fetomaternal alloimmune thrombocytopenia: a UK national study using three data sources. Br J Haematol 2011;152:460-468.
20 Kjeldsen-Kragh J, Killie MK, Tomter G, Golebiowska E, Randen I, Hauge R, Aune B, Oian P, Dahl LB, Pirhonen J, Lindeman R, Husby H, Haugen G, Gronn M, Skogen B, Husebekk A: A screening and intervention program aimed to reduce mortality and serious morbidity associated with severe neonatal alloimmune thrombocytopenia. Blood 2007;110: 833-839.

21 Turner ML, Bessos H, Fagge T, Harkness M, Rentoul F, Seymour J, Wilson D, Gray I, Ahya R, Cairns J, Urbaniak S: Prospective epidemiologic study of the outcome and cost-effectiveness of antenatal screening to detect neonatal alloimmune thrombocytopenia due to anti-HPA-1a. Transfusion 2005;45: $1945-$ 1956. 